\title{
Chapter 6 \\ Standardization of the PCR \\ technique for the detection of \\ bacterial pathogenic. microorganisms inwater using as a model Salmonella spp. and E. coli.
}

\author{
Jose Luis Villarreal Camacho \\ Universidad Libre Seccional Barranquilla. Pathological Biochemistry \\ Research Group \\ jvillarreal@unilibrebaq.edu.co
}

\begin{abstract}
Alfonso Carlos Bettin Martinez
Universidad Metropolitana, Caribbean Research Group. abettinm@unimetro.edu.co
\end{abstract}

\begin{abstract}
Traditional methods for the microorganisms identification are based on the use of strategies such culture media, isolation in selective media, biochemical identification and serological characterization. These strategies are expensive, have low specificity, low sensitivity and are time consuming. In recent years, different molecular strategies such as the Polymerase Chain Reaction (PCR) have been developed, especially quantitative real-time PCR (qPCR) for Salmonella spp. and Escherichia coli detection and quantification in different matrices. These molecular methods are simple, reproducible, flexible and allow a rapid identification. This chapter presents an analysis about methods for Salmonella spp. and E. coli detection, and make emphasis on the use of qPCR and its standardization aimed at the diagnosis of these public health importance microorganisms.
\end{abstract}


Keywords: Salmonella spp., Escherichia coli, qPCR, Foodborne diseases.

Research project which this chapter derives: Molecular detection of difficult isolation microorganisms.

Source of funding: Universidad Libre Seccional Barranquilla.

\section{INTRODUCTION}

The traditional microbiological techniques used for microorganisms detection applied in most laboratories are based on the phenotypic characteristics identification such as microscopic morphology, reaction to various types of staining, antibiotic susceptibility profile, colonies morphology and biochemical preferences. About this last case, it can be presented the fact that could exist different isotypes of the same isolated strain because the microorganisms biochemical characteristics are not totally stable or they can be influenced by genetic regulation, technical manipulation and by the loss or gain of a plasmid. However, in some cases microorganisms are not detectable in matrices that have a low number of cells (100), because they have been subjected to a high degree of injury that decreases the bacterial cells number in the applied technological processes in the food production or human drinking water processing.

In recent years, the molecular techniques application has initiated a revolution in the diagnosis and monitoring of infectious diseases. These techniques include DNA identification without prior amplification such as biochips (101), DNA probes (102), restriction enzymes and ribotyping. On the other hand, there are DNA identification techniques, which involve previous genetic material amplification such as DNA sequencing, the analysis of Restriction Fragments Length Polymorphisms (RFLP), PCR among others (103). 
of all the aforementioned techniques, PCR is the most widely used in vitro amplification system, it's the main objective to obtain millions of copies from one or several target DNA fragments; is a flexible, reproducible, fast, sensitive and specific technique; which makes it the first alternative for the molecular detection of microorganisms in different types of samples without the culture media need, is also useful for the rapid detection of non-cultivable or demanding microorganisms, to detect variations at species and/or subspecies level, to determine viral load and the detection of genes or gene mutations responsible for drug resistance.

Since its discovery (104), different variations have been introduced to the technique, for example the Multiplex Polymerase Chain Reaction (MPCR), in which different types of DNA are detected in the same reaction; Coupled PCR to a previous reverse transcription reaction (RT-PCR), in which the starting nucleic acid is RNA that is retrotranscribed to DNA with the reverse transcriptase enzyme aid (105). Another widely used variant is quantitative real-time PCR (QPCR), which not only allows simultaneous and continuous detection of fluorescent signals as DNA fragments are amplified, but DNA species can be quantified in order to make accurate assessments of the microorganisms found in a given sample (106).

In a qPCR must be taken into account the factors that influence the process success. Therefore, it is necessary to find the best conditions to optimize the process (107). The factorial analysis is an excellent option to determine the optimal conditions for nucleic acids efficient amplification, since it seeks to reduce the number of tests by grouping the variables that correlate with each other, commencing from a large number of homogeneous variables.

This chapter explores the use of qPCR and its respective standardization for Salmonella spp. and Escherichia coli detection, which are important public health microorganisms. 


\section{REAL-TIME QUANTITATIVE POLYMERASE CHAIN REACTION (qPCR)}

Since its creation in 1990, PCR has become the most widely used molecular technique in the world and its impact on the medical diagnostic world has been profound. Due to the high cost of the PCR system (thermocycler, electrophoresis chamber, photodocumentation system, etc.), the use of specialized locations and highly trained staff, the contamination associated with poor management and use of reagents before and after the amplification, a new PCR variant was developed that uses probes labeled with fluorochromes for amplicons detection, denominated Quantitative Real Time PCR (qPCR). This new technological development eliminated analysis contamination by developing all the reaction and quantification in the PCR tube without opening (108). In microbiology, qPCR is used for the purpose of detecting microorganisms (absence/presence), microbial quantification or microbial load, genotyping or microbial characterization and gene expression profiles.

The PCR typically employs a thermostable enzyme, a pair of oligonucleotides per fragment to be amplified which must be at a XXXpM concentration, magnesium in a range from 2.5 to $3.5 \mathrm{mM}$, triphosphate deoxynucleotides at 200nM and a buffer that favors the enzymatic action by avoiding the drastic change of $\mathrm{pH}$ and ionic strength. This new variant employs a non-intercalating dye that binds to double-stranded DNA and is non-specific as SYBR, which is excited at $488 \mathrm{~nm}$ and emits light at $522 \mathrm{~nm}$ (green color) when DNA molecules are amplified. Also, a probe labeled with a fluorochrome is used at one end and a quencher at the other end that absorbs the frequency that the fluorochrome emits, this format is known as Fluorescence Resonance Energy Transfer (FRET) (109). These two molecules interact with each other maintaining a low fluorescence emission.

At the time of executing the thermal protocol, the labeled probe is located 

microorganisms in water using as a model Salmonella spp. and E. coli.

in the region of interest by complementarity and between the pair of oligonucleotides and is displaced by the thermooresisting enzyme system, the probe deoxynucleotide which is labeled when is being displaced emits fluorescence with greater force because it is not near the quencher molecule. The fluorescence must be collected simultaneously and continuously by the optical system of a thermocycler modified for this purpose. These devices are currently coupled to a monitor that graphs the number of cycles against the Relative Fluorescence Units (RFU) or emitted fluorescence numerical values. It should be noted that many people believe that RT-PCR stands for Real Time PCR and is called real-time PCR because it can see the fluorescence as soon as it is emitted. A lie because at beginning the first computers were not coupled to a monitor.

In fact, it is called quantitative PCR because it allows us to count the generated amplicons number at the end by the use of a calibration curve.

As you can see there are many factors in a qPCR that must be taken into account and that influence its success. Therefore it is necessary to find the best conditions to optimize the process.

- Microorganisms concentration: The presence of microorganisms such as Salmonella spp. and E. coli is not accepted in drinking water, therefore the purpose of concentrating microorganisms is to increase the probability of finding them. They are in a homogeneous distribution in the matrix of interest and concentrating them in the same unit of area has as an objective to increase the detection limit and guarantees a good amount of nucleic acids. Centrifugation is the most commonly used method, especially in water. At the end of this process there is a precipitate at the bottom of the tube known as biomass to which a nucleic acid extraction is carried out.

- Another well-used method is filtration (110) with or without centrifuga- 
tion and is based on the difference in microorganism's size to separate or trap them either in the membrane or filter or at the bottom of the tube. The use of a centrifuge prevents the use of a vacuum pump. In the case of solid matrices as food, non-selective enrichment culture media are employed which seek to increase the number of microorganisms by providing them with adequate growth conditions, are subsequently trapped by the aforementioned methods and subjected to nucleic acid extractions.

- DNA or RNA obtaining: Many kits for nucleic acids extraction from different types of samples are currently on the market. They are based on bacterial lysis through the use of enzymes, homogenization, sonication, or mechanical lysis with glass beads; followed by proteins selective precipitation with salts or organic solvents and finally a nucleic acids precipitation with ethanol or isopropanol. Regardless of the type of DNA or RNA extraction, quality and concentration play a key role. The kits have good results, however they are expensive and a good alternative is the use of the organic technique for DNA extraction with phenol-chloroform-isoamyl alcohol accompanied by a previous enzymatic lysis with lysozyme A. A good storage condition to avoid the fractionation, is to store the DNA or RNA at $-80^{\circ} \mathrm{C}$, this makes available those nucleic acids for future studies or doubtful result verification.

- Nucleic acids quantification: Although qPCR is capable of producing millions of amplicons from a low initial copy numbers, the nucleic acids concentration is particularly important. To determine the concentration and purity, spectrophotometry should be used at $260 \mathrm{~nm}$ for nucleic acids and at $280 \mathrm{~nm}$ for proteins, since after typical nucleic acids extraction DNA or RNA is usually available in sufficient quantity and with a high purity. For cases, where impurities in the DNA obtained from a PCR are suspected, fluorometry is recommended as the quan- 
tification method. The dilution of the nucleic acids must be taken into account in the calculation and must be performed with the same water in which the nucleic acids have been rehydrated after the treatment to precipitate with ethanol or isopropanol. If there is little sample, it is advisable to use $1 \mathrm{~cm}$ cells of light path but with final volumes as small as $200 \mu \mathrm{L}$ or to use spectrophotometers that use micro volumes of samples. Relative quantification with imaging systems may be a valid alternative as well.

- Oligonucleotides: after choosing a good pair of oligonucleotides, an important recommendation is to briefly centrifuge the tubes before opening them. They come lyophilized and the gloves electrostatic can attract them and remove them from the tube, since in the shipment they suffer from many movements. The use of talc-free gloves is also recommended. The oligonucleotides must be rehydrated in $10 \mathrm{mM}$ Tris $\mathrm{pH} 8.0$ and $1 \mathrm{mM}$ EDTA (TE). Other users prefer DNAsas and RNAsas water free which is also a good strategy. Whether water or TE is recommended to make a stock solution at a concentration of $100 \mu \mathrm{M}$, this is achieved by adding ten times the amount of water or TE of the oligonucleotide nanomoles. From this solution a work solution must be made, which must be at a concentration of $10 \mu \mathrm{M}$ and its final volume will be according to the number of samples to be analyzed; this last solution will be the one used in the reactions or production of mastermix. Genes such as hilA, invasiveness regulatory gene (111); ompF, outer membrane porin f; $t t r$, tetrathionate reductase enzyme (112); iagA, gene activator of invasion genes expression (113); ssaN, type III secretion system of ATP synthetase (114) are frequently amplified for these pathogens detection.

- Probes: The same recommendations should be followed as with oligonucleotides but additionally because they are labeled with fluorochromes they should be worked in darkness as their activity may 
be lost. The work solutions that will be subjected to several thawing must be storage in amber color tubes.

- Thermocycler: Most equipment uses plastic tubes and lids to collect fluorescence, however a few use glass tubes. An important aspect is that last generation equipment allows finding experimentally oligonucleotides best alignment temperature, by the application of thermal protocols with temperatures of gradient, which means that from a set range the equipment is able to apply different temperatures per row, making faster the standardization process. Such temperature is calculated from the information of the melting temperature or Tm which is in the information sheet of the oligonucleotides. A good recommendation is to acquire brands that can work with different types of chemistry and have the ability to turn off data collection channels so that they can be used as conventional thermocyclers to increase the life of the optical system.

- Electrophoresis: Although many do not find it convenient, electrophoresis plays a fundamental role in a standardization process. The direct verification of the expected bands, weak bands or be able to discard nonspecific bands become a means of validation in the standardization early stages.

- Positive controls: the use of a known DNA or RNA that always produces an amplification signal that validates and gives confidence about obtained results. Many people believe that an ideal positive control in the case of Salmonella spp. or Escherichia coli detection in water would be a DNA or RNA sample from feces of a sick individual. In this particular case, an ideal positive control is the DNA or RNA of a strain isolated from these microorganisms mentioned, since it will always show an amplification signal. No signal with the positive control is signal is 

microorganisms in water using as a model Salmonella spp. and E. coli.

indicative of an error with the reagents (calculations, expiration dates) or equipment malfunction.

- Blank: Usually confused with a negative control, however in a target water is used instead of nucleic acids. Detecting a amplification signal in a target is indicative of poor technique, cross-contamination or cross-sampling. In all cases where it is used, there should be no amplification signal.

- Endogenous control: Not always used. However, in cases where the microorganism is obtained from tissue of an individual, it is known that there is also DNA or RNA of the subject, therefore a qPCR which detects a constitutive human or animal gene and that is read in another channel increases the technical rigor of a qPCR and increase confidence in the obtained results.

\section{FACTORIAL ANALYSIS}

The factorial design is an excellent option to standardize, finding the best amplification conditions in a qPCR because it allows to examine the effect of different factors or variables on one or several quality indicator responses and, in addition, it facilitates to determine the possible combinations or treatment of the different levels of these factors to finally select that combination of variables generating the desired effect, such as the most efficient amplification that allows to detect the microorganisms of interest.

The factorial analysis is performed with different statistical packages and consists, in this case, of three phases:

- The planning or a matrix design in which all variables and their different levels are combined: for example, many factors influence a qPCR success, but the hybridization temperature of the oligonucleotides 
and magnesium concentration, constitute the most main points in a standardization process. Then what is wantedinthisfirstphaseistodesign a matrix in which they confront the two variables of oligonucleotides hybridization temperature and magnesium concentration including their levels, that is to say, the different magnesium concentrations and the different hybridization temperatures.

- Reduction of data by default of those repeated data: when facing a factorial design with two variables and five levels for the hybridization temperature $\left(5{ }^{\circ} \mathrm{C}\right.$ below the lowest melting temperature of the pair of oligonucleotides to be tested, assuming a $\mathrm{Tm}$ of $58^{\circ} \mathrm{C}$, suggested points are $56{ }^{\circ} \mathrm{C}, 55{ }^{\circ} \mathrm{C}, 54{ }^{\circ} \mathrm{C}, 53{ }^{\circ} \mathrm{C}$ and $52{ }^{\circ} \mathrm{C}$ ) and four levels for magnesium concentration ( $2.5 \mathrm{mM}, 3 \mathrm{mM}, 3.5 \mathrm{mM}$ and $4 \mathrm{mM})$, what is being proposed is a $5 \mathrm{X}_{4}$ factorial design (Table 6.1.)

Table 6.1.

Data matrix or variable combination and their respective levels

\begin{tabular}{|c|c|c|c|c|c|}
\hline \multirow{3}{*}{$\begin{array}{l}\text { Magnesium } \\
\text { concentration }\end{array}$} & \multicolumn{5}{|c|}{$\begin{array}{l}\text { Example of a factorial analysis matrix } 5 \times 4 \text { - for a qPCR } \\
\text { standardization }\end{array}$} \\
\hline & \multicolumn{5}{|c|}{ Hybridization temperature } \\
\hline & $56^{\circ} \mathrm{C}$ & $55^{\circ} \mathrm{C}$ & $54^{\circ} \mathrm{C}$ & $53^{\circ} \mathrm{C}$ & $52^{\circ} \mathrm{C}$ \\
\hline \multicolumn{6}{|l|}{$2.5 \mathrm{mM}$} \\
\hline \multicolumn{6}{|l|}{$3 \mathrm{mM}$} \\
\hline \multicolumn{6}{|l|}{$3.5 \mathrm{mM}$} \\
\hline $4 \mathrm{mM}$ & & & & & \\
\hline
\end{tabular}

In the given example what is drawn or omitted are those combinations which are repeated and which will not be carried out experimentally. The obtained results are entered as numerical results. For the case of the qPCR, these values may be absolute such as the threshold point or CT or relative, for example when comparing the results with a positive control which is assumed to have no variation. A valuable alternative in the early stages of experimentation is to feed the computer program with data from an image analysis obtained with a photodocumentator. This strategy although a 
little expensive will give us a record and evidence of bands presence or unwanted bands.

- Optimization of analysis: this phase directs the computer program with the different statistics that are desired to calculate and thus facilitate future results interpretation.

\section{DISCUSSION}

Many authors have devoted themselves to standardize the qPCR techniques emphasizing the concentration time or microorganisms pre-enrichment, optimizing the detection of Salmonella spp. in 12 hours, which includes 8 hours of pre-enrichment, followed by an genetic material automatic extraction and finally the molecular detection. Different nutrient media such as peptone water, BHI broth and soy trypticase broth were evaluated, however, no significant differences were found in any of the applied tests (115).

In another assay conducted by D'Ursoa and collaborators, who evaluated a different concentration method, which consisted of filtration for the viable bacteria recovery of Salmonella enterica and Listeria monocytogenes in 30 minutes. The filtration procedure consisted of passing the bacterial suspension on a $0.2 \mu \mathrm{m}$ diameter filter paper for Salmonella enterica and $0.4 \mu \mathrm{m}$ for Listeria monocytogenes. This treatment showed no effect on the viability of the cells. In the case of food the technique was able to detect 10 bacteria per $10 \mathrm{~g}$ of Yogurt and also able to detect viable cells in the presence of a wide range of dead cells (116).

Another way to standardize is to evaluate the selectivity that includes oligonucleotides and probes inclusivity and exclusivity in qPCR assays, since they are important elements for the design of the diagnostic methodology, such as chen and collaborators. They designed the oligonucleotides to amplify a sequence within the ATP synthetase gene of the type III secretion 
system (ssaN), and designed an internal amplification control with their respective probe. The assay demonstrated $100 \%$ inclusivity for the 40 Salmonella strains tested and $100 \%$ exclusivity for the 24 unrelated strains (114).

\section{CONCLUSION}

Strict quality control of reagents, preventive maintenance of equipment and locations, technical rigor of molecular tests, biosecurity precautions and standards, good oligonucleotides and probes selection along with a statistical methodology, make the qPCR a superior tool in the pathogenic microorganisms' detection.

Costs for the microorganisms' molecular detection such as Salmonella spp. and E. coli, are high compared to traditional microbiological methods, but the benefits to the health sector by achieving a rapid and accurate diagnosis due to its high sensitivity and high specificity and the cost benefit ratio that gives to the productive sector by allowing the food products release to the market more quickly justify the implementation of this technique.

New methods and combinations of techniques make the detection process a less cumbersome, fast, sensitive and specific procedure, but the need to perform the confirmation by the traditional method is still valid and mandatory according to the legislation in some countries. These techniques have allowed discriminating between living or dead microorganisms, active or inactive molecules, allowing carrying out sanitary controls. The qPCR for Salmonella spp. and E. coli will complement but not replace traditional microbiological techniques that are necessary for epidemiological surveillance purposes.

How to quote this chapter:

Villarreal Camacho JL, Bettin Martínez AC. Standardization of the pcr technique for the detection of bacterial pathogenic microorganisms in water using as a model Salmonella spp. and E.coli. In Badillo Viloria M, Pérez Lavalle L, editors. Realtime PCR applied to bacterial waterborne pathogens detection and quantification. Barranquilla: Ediciones Universidad Simón Bolívar; 2018. p. 77-88. 